\title{
Different Imaging Features of Retroperitoneal Unicentric Castleman's Disease: A Case Report
}

\author{
Yonghong Cao ${ }^{a}$ Erlan Shi ${ }^{a}$ Jun Ye ${ }^{a}$ Rong Zhang ${ }^{a}$ Yunsheng Wang ${ }^{a}$ \\ Mao Sheng ${ }^{b}$ Zhuping Su ${ }^{b}$ Wu Dai ${ }^{a}$ \\ aDepartment of Endocrinology, Hefei Hospital affiliated to Anhui Medical University \\ (The Second People's Hospital of Hefei), Hefei, China; ${ }^{b}$ Department of Imageology, Hefei \\ Hospital affiliated to Anhui Medical University (The Second People's Hospital of Hefei), \\ Hefei, China
}

\author{
Keywords \\ Lymphadenopathy · Castleman's disease · Unicentric Castleman's disease · Retroperitoneal \\ tumor $\cdot$ Imaging features
}

\section{Abstract}

Castleman's disease is a rare disease which is difficult to diagnose early due to its lack of specific manifestations, and also is easily confused with lymphoma or other solid tumors. Castleman's disease can occur in any part of the body containing lymph nodes and is most common in the chest, followed by the neck, abdomen, and axillae. A 37-year-old woman was admitted to our hospital because of a tumor near the adrenal gland found by computed tomography. Positron emission tomography-computed tomography revealed that the retroperitoneal tumor may be a malignant disease. However, the pathological diagnosis after laparoscopic resection was retroperitoneal Castleman's disease, hyaline vascular type.

(C) 2020 The Author(s).

Published by S. Karger AG, Basel

\section{Introduction}

Castleman's disease (CD), also known as giant lymph node hyperplasia, is a benign lymphoproliferative disorder affecting both lymph nodes and extranodal loci [1]. Benjamin Castleman first reported a rare benign lymphadenoproliferative disease in 1954 [2]. CD can occur in any part of the body containing lymph nodes and is most common in the chest, 


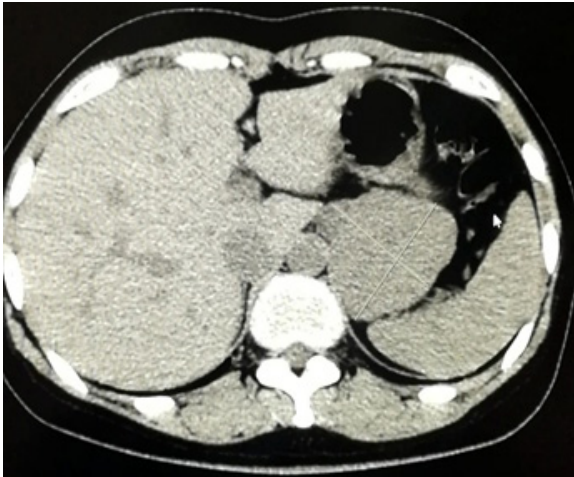

$\mathrm{a}$
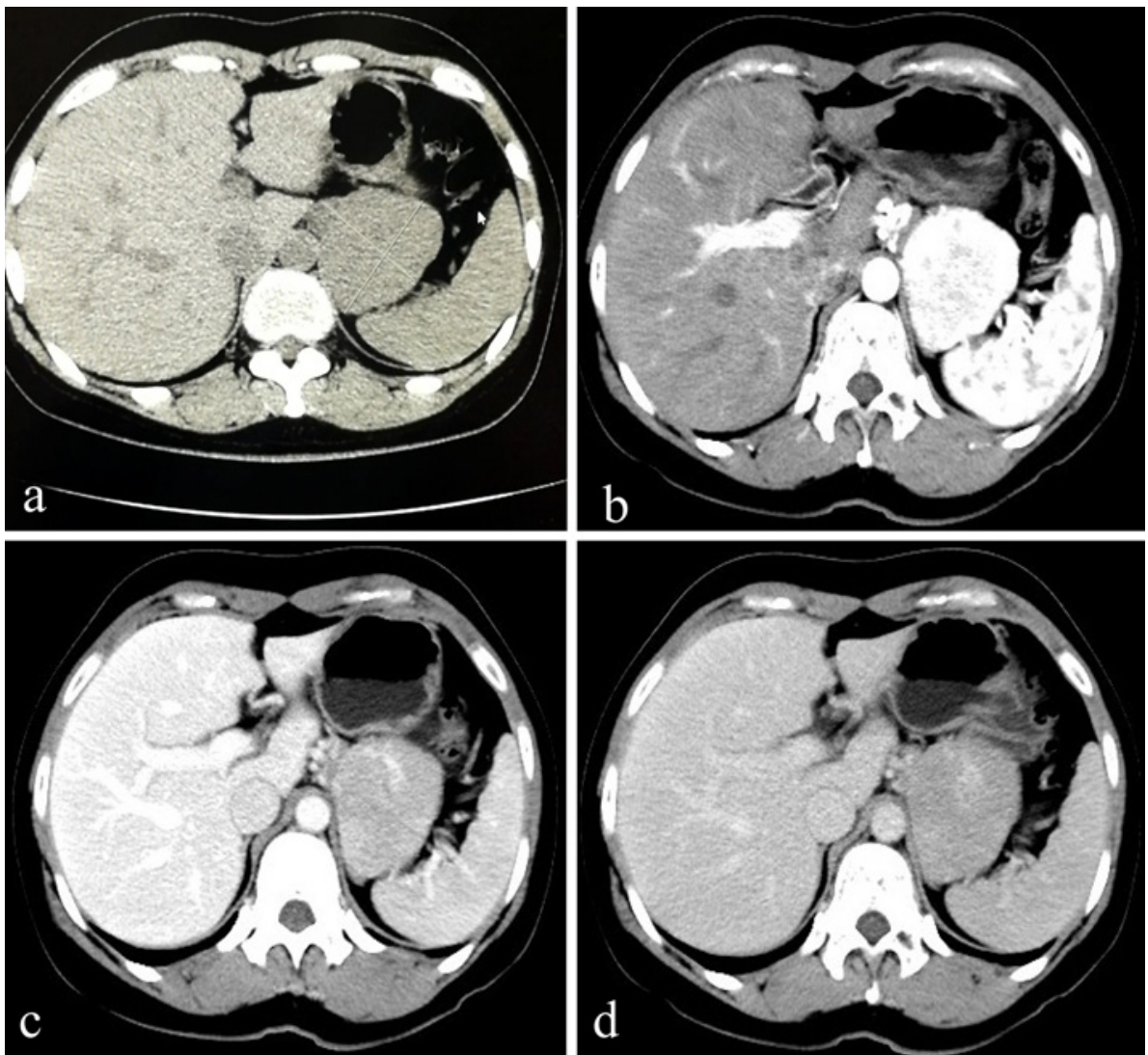

Fig. 1. Computed tomography images of the patient. a Nonenhanced computed tomography image. b Arterial phase. c Venous phase. d Delayed phase.

followed by the neck, abdomen, and axillae $[3,4]$. However, retroperitoneal CD is relatively rare. We report on a patient with retroperitoneal CD who presented no clinical symptoms; only by computed tomography (CT) was a large abdominal tumor found.

\section{Case Presentation}

A 37-year-old woman was admitted to our hospital because of a tumor near the adrenal gland, which was found at a periodic health checkup by CT. The CT scan showed that the tumor was about $6 \times 5 \mathrm{~cm}$ in size, near the adrenal gland, with uniform density and no ischemic necrosis (Fig. 1a).

She had no disease history or family history. She did not have any other clinical symptoms such as hypertension, headache, hot flashes, feebleness, low fever, night sweats, vomiting, etc. Laboratory data showed that almost all findings were normal, including her adrenal hormone level, and she was HIV negative, apart from a slight elevation of CA 19-9 47 U/mL (reference, 0 -35.0). Positron emission tomography-CT (PET-CT) revealed that the tumor had a maximum standardized uptake value (SUV $\mathrm{Sax}_{\max }$ ) of 11.8 (Fig. 2a, b), and a delayed imaging $\mathrm{SUV}_{\max }$ of 13.6 (Fig. 2c, d), which suggested malignant disease or CD.

The patient was referred to our hospital for an enhanced CT and computed tomography angiography (CTA) examination. The enhanced CT values for the arterial phase, venous phase, 

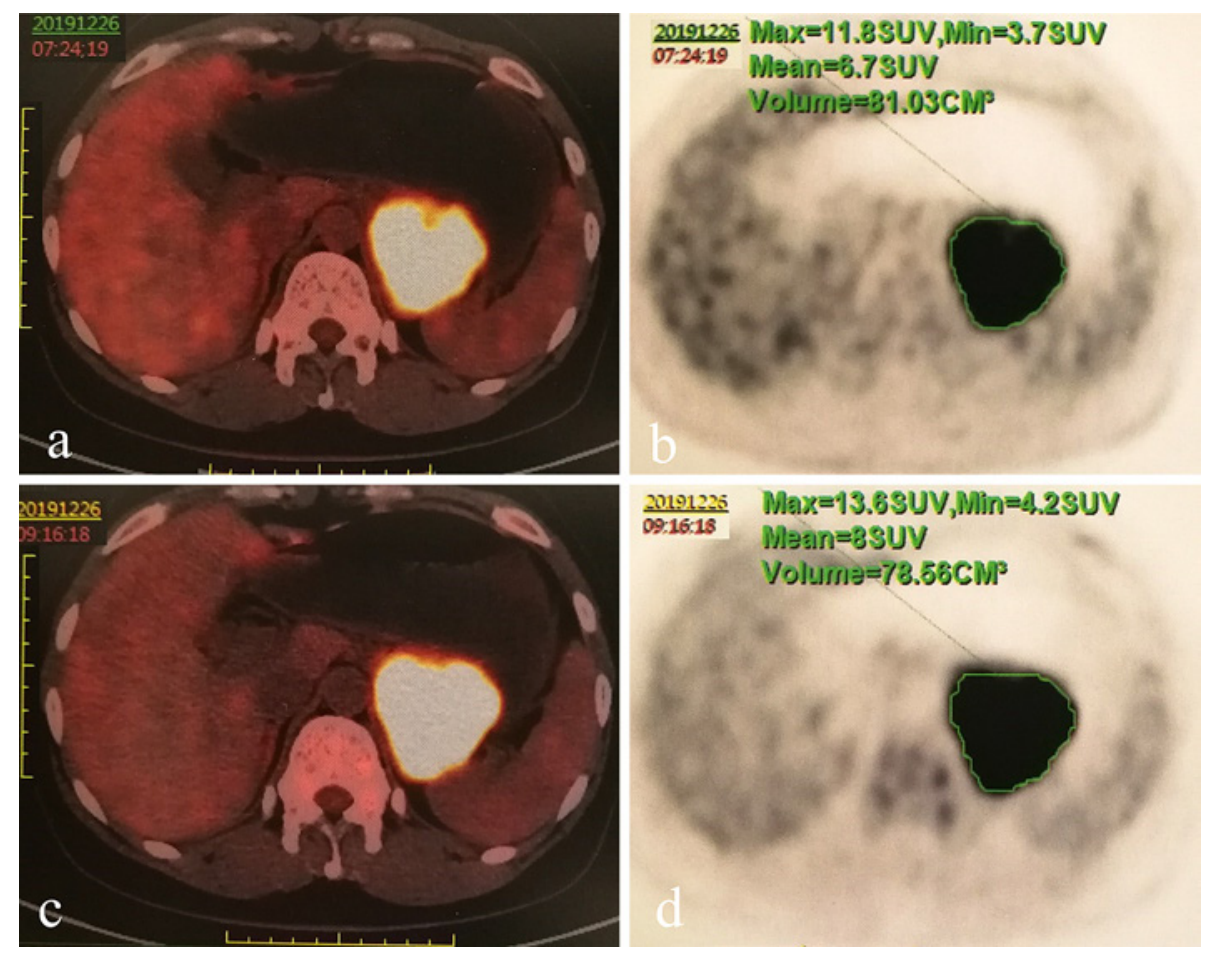

Fig. 2. Positron emission tomography-computed tomography images. a, b Standardized uptake value $\left(\mathrm{SUV}_{\max }\right) . \mathbf{c}$, d Delayed imaging $\mathrm{SUV}_{\max }$.

and delayed phase were 164, 97, and $82 \mathrm{HU}$ (Fig. 1b-d). The results of CTA showed that the blood supply to the retroperitoneal mass was abundant, mainly from the left gastric artery, combined with an arteriovenous fistula (Fig. 3).

The possibility of retroperitoneal CD was then considered. Finally, laparoscopic resection of retroperitoneal tumorectomy was performed. The resected specimen was gray-white and soft, with a size of $80 \times 60 \times 40 \mathrm{~mm}$, and covered with a capsule. HE (hematoxylin and eosin) staining showed nodular hyperplasia and atrophy of the germinal center, whereas the proliferation of the peripheral lymphocytes was arranged in an "onion skin" pattern (Fig. 4a). The immunohistochemistry results were as follows: CD20+, CD79a+, CD10-, Bcl-6-, Bcl-2+, IgD+, and cyclin D1- (Fig. 4b). Based on the lack of any other lymph node enlargement and the above examination and histopathological results, CD was diagnosed, subclassified as the hyaline vascular type (HV).

\section{Discussion}

CD is a rare disease which is difficult to diagnose early due to its lack of specific manifestations, and also is easily confused with lymphoma or other solid tumors. There are two types of CD in the clinic: unicentric CD (UCD) and multicentric CD (MCD). According to pathological results, it is divided into three types: HV, plasma cell type (PC), or mixed type [5].

Because of quite different clinical features and prognoses, UCD and MCD are considered to be two distinct diseases. In the UCD group, patients are younger (median age, 33 years; the age of the patient we reported on was 37 years) and most cases have no clinical symptoms (the same as in our case). UCD, in particular, is often an unexpected discovery during routine 

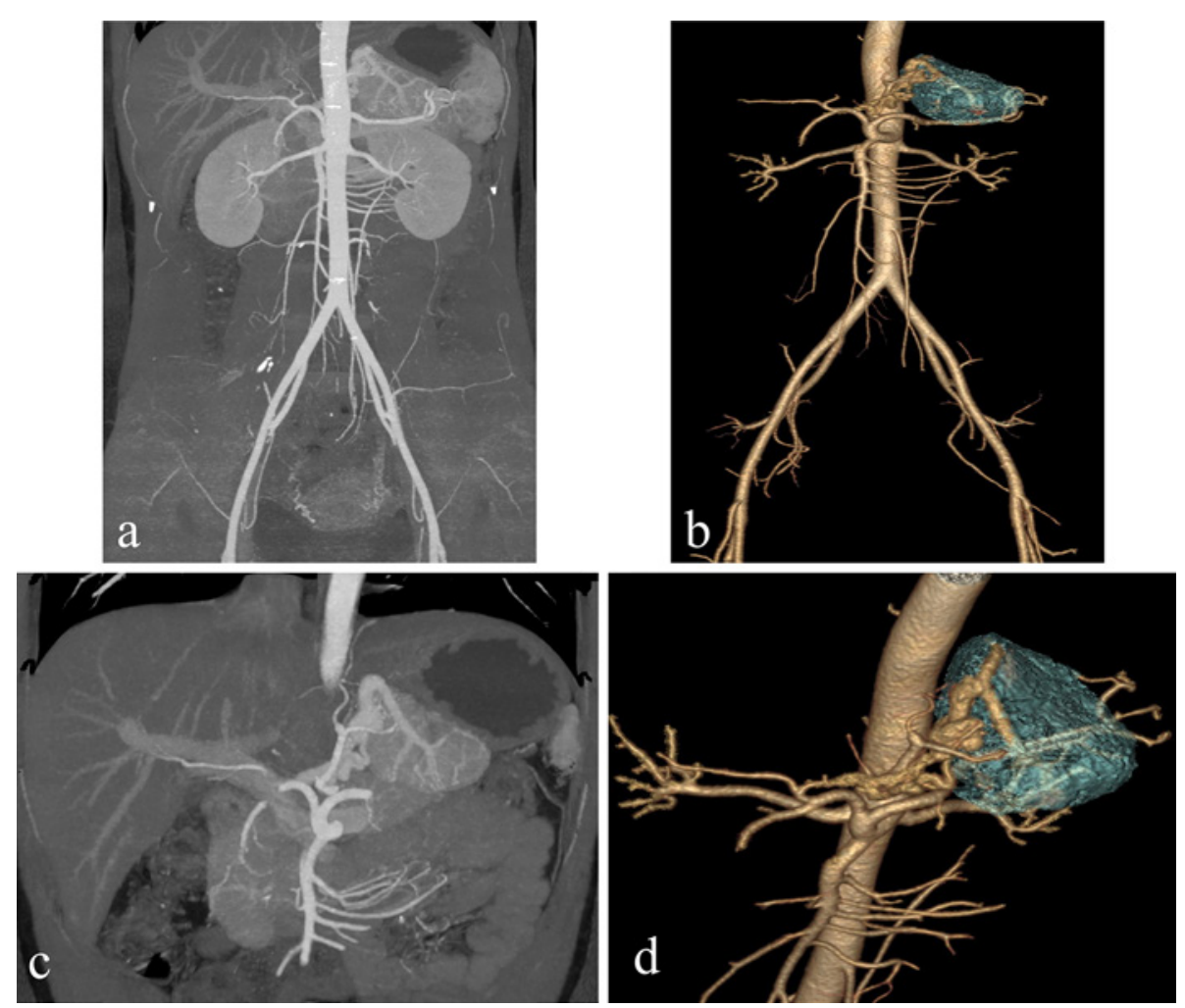

Fig. 3. Computed tomography (CT) angiography images. a, c Maximum intensity projection with spiral CT. b, d Volume rendering with spiral CT.
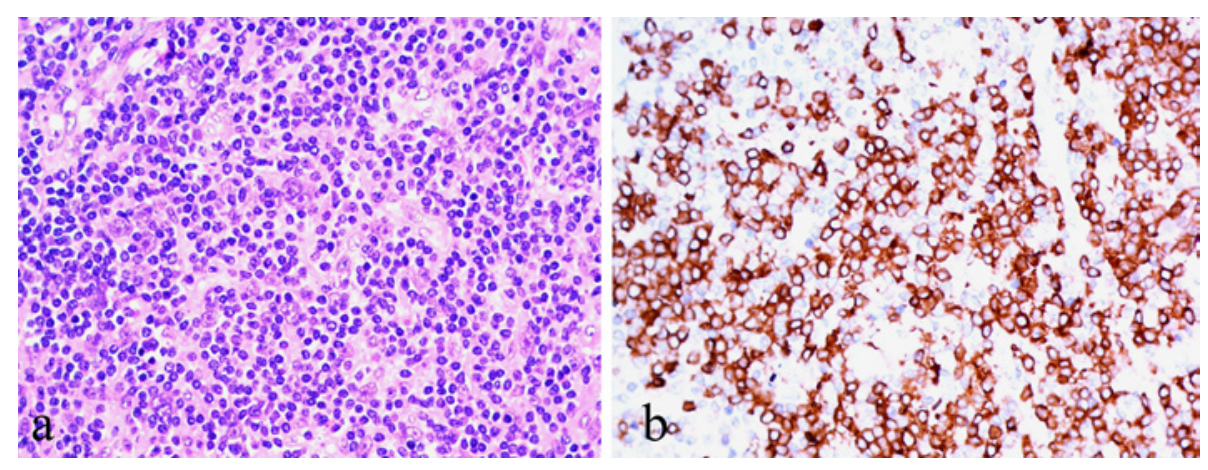

Fig. 4. HE (a) and immunohistochemistry (b) for CD79a.

examination. Most commonly, it is accompanied only by local lymphadenopathy. In contrast, MCD can manifest with a very serious hypercytokinemia-driven inflammatory syndrome mostly caused by interleukin-6 [6]. The most common histological type of UCD is HV, accounting for $91.7 \%$ of UCD cases [5]. It is identified in most of the possible locations found in other case reports, confirming that HV UCD has no limits regarding localization [7, 8]. In contrast, most of MCD patients have significant clinical symptoms, and the histopathological subtype is PC [9].

What are the characteristics of $\mathrm{CD}$ on imaging? Most of the lesions encountered are noninvasive masses with well-defined borders. Use of nonenhanced or enhanced CT scanning 
is related to the location and pathological type of the tumor. On nonenhanced CT, the case of retroperitoneal $\mathrm{CD}$ that we reported on was hypodense to isodense relative to skeletal muscle, with uniform density and no ischemic necrosis. The enhancement characteristics of CD are closely related to its pathological type. In our case, enhanced CT showed that there were abundant hyperplastic capillaries and peripheral blood supply vessels in the HV. In addition, on a multiphase contrast-enhanced scan, it was characterized by significant or obvious enhancement in the arterial phase, portal venous phase, and delayed phase, and by being "early in and late out." Zhao et al. [10] found that more than half of their patients had hypertrophied vessels on CT images; moreover, they all had HV CD. Furthermore, the degree of enhancement was not significantly different between the patients with HV CD and those with PC CD [10-12]. In our case, CTA showed that the blood supply to the retroperitoneal mass was abundant, which might explain the high uptake of the mass on PET-CT. Zhao et al. [10] also found that calcification was observed in all the patients with HV UCD; thus, they thought that calcification and hypertrophied vessels may be valuable diagnostic features of HV UCD.

For UCD, complete resection of the involved lesion is considered the gold standard treatment, and recurrent cases are rare [13]. It has been curative in almost all cases reported so far, with a 5-year overall survival rate approaching 100\% [14]. However, unlike for UCD, an optimal treatment for MCD has not been well established, and the outcome is less favorable.

CD is extremely rare, so it is necessary to improve the awareness of its diagnosis. It is helpful to refer to imaging results for the diagnosis of $\mathrm{CD}$ and make treatment plans based on pathological results. We report on this case of retroperitoneal UCD in order to improve clinicians' understanding of $\mathrm{CD}$ and provide reference for future diagnosis.

\section{Statement of Ethics}

Written informed consent to participate and for publication was obtained from the patient. A copy of the written consent form is available for review from the editor-in-chief of this journal. This article was approved by the Ethics Committee of Hefei Hospital affiliated to Anhui Medical University.

\section{Disclosure Statement}

The authors declare that they have no competing interests.

\section{Funding Sources}

No funding was received.

\section{Author Contributions}

Cao Yonghong and Shi Erlan drafted the manuscript; Wang Yunsheng provided the clinical information; Sheng Mao and Su Zhuping made the imaging diagnosis; Dai $\mathrm{Wu}$, Ye Jun, and Zhang Rong participated in manuscript revision. 


\section{Availability of Data and Material}

Due to ethical restrictions, the raw data underlying this paper are available upon request to the corresponding author.

\section{References}

1 Wu D, Lim MS, Jaffe ES. Pathology of Castleman disease. Hematol Oncol Clin North Am. 2018 Feb;32(1):37-52.

2 Case records of the Massachusetts General Hospital Weekly Clinicopathological Exercises: case 40011. N Engl J Med. 1954 Jan;250(1):26-30.

3 Kligerman SJ, Auerbach A, Franks TJ, Galvin JR. Castleman disease of the thorax: clinical, radiologic, and pathologic correlation: from the radiologic pathology archives. Radiographics. 2016 Sep-0ct;36(5):1309-32.

4 Jiang XH, Song HM, Liu QY, Cao Y, Li GH, Zhang WD. Castleman disease of the neck: CT and MR imaging findings. Eur J Radiol. 2014 Nov;83(11):2041-50.

5 Zhang X, Rao H, Xu X, Li Z, Liao B, Wu H, et al. Clinical characteristics and outcomes of Castleman disease: a multicenter study of 185 Chinese patients. Cancer Sci. 2018 Jan;109(1):199-206.

6 Yoshizaki K, Murayama S, Ito H, Koga T. The role of interleukin-6 in Castleman disease. Hematol Oncol Clin North Am. 2018 Feb;32(1):23-6.

7 Oksenhendler E, Boutboul D, Fajgenbaum D, Mirouse A, Fieschi C, Malphettes M, et al. The full spectrum of Castleman disease: 273 patients studied over 20 years. Br J Haematol. 2018 Jan;180(2):206-16.

8 van Rhee F, Greenway A, Stone K. Treatment of idiopathic Castleman disease. Hematol Oncol Clin North Am 2018 Feb;32(1):89-106.

9 Dispenzieri A, Armitage JO, Loe MJ, Geyer SM, Allred J, Camoriano JK, et al. The clinical spectrum of Castleman's disease. Am J Hematol. 2012 Nov;87(11):997-1002.

10 Zhao S, Wan Y, Huang Z, Song B, Yu J. Imaging and clinical features of Castleman disease. Cancer Imaging. 2019 Jul 25;19(1):53.

11 Hill AJ, Tirumani SH, Rosenthal MH, Shinagare AB, Carrasco RD, Munshi NC, et al. Multimodality imaging and clinical features in Castleman disease: single institute experience in 30 patients. Br J Radiol. 2015 May; 88(1049):20140670.

12 Kwon S, Lee KS, Ahn S, Song I, Kim TS. Thoracic Castleman disease: computed tomography and clinical findings. J Comput Assist Tomogr. 2013 Jan-Feb;37(1):1-8.

13 Shimokihara K, Kawahara T, Kasahara R, Kasuga J, Sugiura S, Tajiri R, et al. Retroperitoneal Castleman's disease. Case Rep Oncol. 2019 Nov;12(3):885-9. http://dx.doi.org/10.1159/000504700.

14 Larroche C, Cacoub P, Godeau P. Castleman's disease [in French]. Rev Med Interne. 1996;17(12):1003-13. http://dx.doi.org/10.1016/s0248-8663(97)80844-2. 